\title{
Supermassive black holes, pseudobulges, and the narrow-line Seyfert 1 galaxies
}

\author{
Smita Mathur ${ }^{2}$ \\ Astronomy Department, The Ohio State University, Columbus, OH 43210 \\ smita@astronomy. ohio-state.edu \\ Dale Fields \\ L.A. Pierce College, Woodland Hills, CA, 91371 \\ FieldsDL@piercecollege.edu \\ Bradley M. Peterson ${ }^{2}$ \\ Astronomy Department, The Ohio State University, Columbus, OH 43210 \\ peterson@astronomy.ohio-state.edu \\ Dirk Grupe \\ Pennsylvania State University, State College, PA \\ grupe@astro.psu.edu
}

\begin{abstract}
We present HST/ACS observations of ten galaxies that host narrow-line Seyfert 1 (NLS1) nuclei, believed to contain relatively smaller mass black holes accreting at high Eddington ratios. We deconvolve each ACS image into a nuclear point source (AGN), a bulge, and a disk, and fitted the bulge with a Sersic profile and the disk with an exponential profile. We find that at least five galaxies can be classified as having pseudobulges. All ten galaxies lie below the $M_{\mathrm{BH}^{-}}$ $\mathrm{L}_{\text {bulge }}$ relation, confirming earlier results. Their locus is similar to that occupied by pseudobulges. This leads us to conclude that the growth of BHs in NLS1s is governed by secular processes rather than merger-driven. Active galaxies in
\end{abstract}

\footnotetext{
${ }^{2}$ Center for Cosmology and Astro-Particle Physics, The Ohio State University, Columbus, OH 43210
} 
pseudobulges point to an alternative track of black hole-galaxy co-evolution. Because of the intrinsic scatter in black hole mass-bulge properties scaling relations caused by a combination of factors such as the galaxy morphology, orientation, and redshift evolution, application of scaling relations to determine $\mathrm{BH}$ masses may not be as straightforward as has been hoped.

Subject headings: galaxies:active - galaxies:nuclei-galaxies:spiral

\section{Introduction}

Active galaxies are "active" because they accrete matter onto their supermassive black holes. However, whether this accretion leads to a significant growth of the nuclear black hole has been a matter of some debate. Results on the X-ray background and the better determination of the local black hole mass density have led to the conclusion that indeed, most of the black hole growth happens during the active phase (e.g. Barger et al. 2001, Aller \& Richstone 2002, Yu \& Tremaine 2002, Graham \& Driver 2007).

The mass of the nuclear black hole, $M_{\mathrm{BH}}$, and the bulge luminosity of its host galaxy appear to be correlated (Magorrian et al. 1998). The correlation between $M_{\mathrm{BH}}$ and the bulge velocity dispersion $\sigma$ is also observed to be very tight with scatter of only 0.3dex (Gebhardt et al. 2000a, Ferrarese \& Merritt 2000, Merritt \& Ferrarese 2001) with $\log M_{\mathrm{BH}}=a+b \times \log$ $\left(\sigma_{*} / \sigma_{0}\right)$ with $M_{\mathrm{BH}}$ in units of $M_{\odot}$ and $\sigma_{0}=200 \mathrm{~km} \mathrm{~s}^{-1}, b=4.02$ and $a=8.13$ (Tremaine et al. 2003). Basically, the mass of the black hole seems to be correlated with the mass of the bulge (Häring \& Rix 2004). Interestingly, the above relation for normal galaxies also extends to active galaxies (Gebhardt et al. 2000b, Ferrarese et al. 2001, McLure \& Dunlop 2002, Woo \& Urry 2002), provided correction factors related to the unknown geometry of the broad emission line region are used.

The results discussed above imply that the formation and growth of the nuclear black hole and the bulge in a galaxy are intimately related, and several theoretical models have attempted to explain the observed $M_{\mathrm{BH}^{-}} \sigma$ and $M_{\mathrm{BH}}-\mathrm{L}_{B u l g e}$ relations (e.g. Haehnelt 2003, Haehnelt et al. 1998, Adams et al. 2001, King 2003). It is of interest, therefore, to follow the tracks of AGNs in the $M_{\mathrm{BH}^{-}} \sigma$ or $M_{\mathrm{BH}^{-}} \mathrm{L}_{B u l g e}$ plane to discriminate among models and so to understand this concurrent growth. Since high accretion rates would lead to substantial black hole growth, active galaxies with close to Eddington accretion are perhaps the best candidates. At low redshift, abundant observational evidence suggests that narrow line Seyfert 1 galaxies (NLS1s; a subclass of Seyfert galaxies, whose most notable feature is that the full widths at half maximum of $\mathrm{H} \beta$ lines are less than $2000 \mathrm{~km} \mathrm{~s}^{-1}$; Osterbrock \& Pogge 
1985; Goodrich 1989) accrete at close to Eddington rate (e.g., Pounds et al. 1995, Grupe 2004 and references therein). NLS1s are found to lie below the $M_{\mathrm{BH}^{-}}$-bulge relations (both $M_{\mathrm{BH}}-\sigma$ and $M_{\mathrm{BH}}-\mathrm{L}_{\text {Bulge }}$ ) of normal galaxies and broad line Seyfert 1s (BLS1s) (Mathur et al. 2001, Czerny et al. 2001, Wandel 2002). Using a complete sample of soft X-ray selected AGNs, this result was confirmed by Grupe \& Mathur (2004; see also Mathur \& Grupe 2005a, 2005b; Watson et al. 2007). The statistical result is robust and is not due to any systematic measurement error. However, it was obtained by using FWHM([OIII]) as a surrogate for the bulge velocity dispersion and the black hole mass was estimated using the

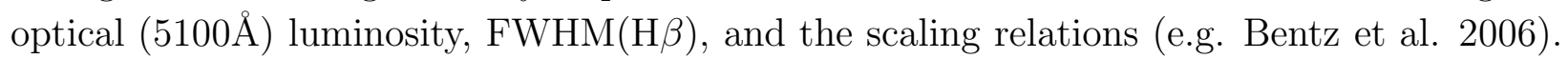
Black hole mass estimates using two other methods gave consistent results, suggesting that the BH masses are not underestimated; Mathur et al. (2001) used accretion disk model fits to estimate BH masses, and Nikolajuk et al. (2009) used variability power density spectra for the purpose. However, the use of $\mathrm{FWHM}([\mathrm{OIII}])$ as a surrogate for velocity dispersion was questioned by many authors. For example, Komossa \& Xu (2007) argued that if the [OIII] line is blueshifted, or if it has asymmetric blue wings, then it ceases to be a good indicator of the bulge velocity dispersion. Therefore, it is necessary to confirm or refute whether NLS1s really lie below the $M_{\mathrm{BH}^{-}} \sigma$ relation. Similarly, it would be useful to make accurate measurements of $\mathrm{L}_{B u l g e}$ and confirm whether NLS1s lie below the $M_{\mathrm{BH}}-\mathrm{L}_{\text {Bulge }}$ relation. If confirmed, it would imply that at low-redshift black holes grow by accretion in well-formed bulges; as they grow, they may move closer to the $M_{\mathrm{BH}}-\sigma$ or $M_{\mathrm{BH}}-\mathrm{L}_{\text {Bulge }}$ relations for normal galaxies. This result, if confirmed, would not support theories of $M_{\mathrm{BH}}-\sigma$ relation in which the black hole mass is a constant fraction of the bulge mass at all times in the life of an AGN or those in which bulge growth is controlled by AGN feedback (as discussed in detail by Mathur \& Grupe 2005b). If, on the other hand, we find that the NLS1s do lie on the $M_{\mathrm{BH}}$-bulge relations, the implications are again interesting. The NLS1s in the sample of Grupe \& Mathur have smaller mass BHs compared to BLS1s; if they all follow the same $M_{\mathrm{BH}}$-bulge relations then it would imply that in the present epoch, highly accreting supermassive black holes exist in smaller galaxies. This will provide important input to our understanding of $\mathrm{BH}$-galaxy co-evolution.

Using an optically selected sample of NLS1s (Williams et al. 2002), Williams et al. (2004) have shown that NLS1 galaxies are a mixed bag. While some show steep X-ray spectra and strong FeII emission, some do not. Those NLS1s which show these extreme properties are highly likely to be accreting at a high Eddington rate and those are the ones which lie below the $M_{\mathrm{BH}^{-}} \sigma$ relation (Mathur \& Grupe 2005b). There are undoubtedly some NLS1s having narrow emission lines as a result of their face-on orientation. The newly discovered $\gamma$-ray active NLS1s observed with Fermi (Abdo et al. 2009) are most likely viewed close to the jet axis, confirming earlier similar conclusions from radio observations 
(Komossa et al. 2006, Yuan et al. 2008). However, as a class, NLS1s are much less radio-loud than BLS1s, arguing against a predominance of beaming and face-on views in NLS1s as a class (Komossa et al. 2006). It is quite possible that these beamed NLS1s are not highly accreting. Our focus here, however, is on objects with evidence for high Eddington accretion.

We performed HST/ACS imaging of host galaxies of a sample of 10 NLS1s that appear not to lie on the $M_{\mathrm{BH}^{-}} \sigma$ relation in Grupe \& Mathur (2004). In principle, we could measure $\sigma$ directly with the CaII triplet line. However, for many of the NLS1s in our sample, the CaII lines fall in the water vapor band in the Earth's atmosphere. In many NLS1s for which CaII line is accessible from ground, CaII is observed in emission rather than in absorption (Rodriguez-Ardila et al. 2002). This makes the use of stellar absorption features to determine $\sigma$ difficult for the targets of interest. The goal of the HST observations was to measure the fundamental plane parameters $\left\langle I_{e}\right\rangle$ and $R_{e}$ and so get an alternative handle on $\sigma$ (the bulge luminosity is related to the stellar velocity dispersion $\sigma_{*}$ through the fundamental plane of galaxies with $\log \mathrm{R}_{e}=1.24 \log \sigma-0.82 \log \left\langle I_{e}\right\rangle-\mathrm{C}$ where $\mathrm{R}_{e}$ is the effective radius of the bulge profile and $\left\langle I_{e}\right\rangle$ is the average surface brightness inside $\mathrm{R}_{e}$ (e.g. Dressler et al. 1987; Djorgovski \& Davis 1987)). We could then plot the loci of our galaxies on both the $M_{\mathrm{BH}^{-}}$ $\mathrm{L}_{B u l g e}$ and $M_{\mathrm{BH}^{-}} \sigma$ planes. However, we show in $\S 4$ that the bulges of at least some of our target galaxies are actually pseudobulges which do not lie on the fundamental plane. As such, we cannot infer $\sigma$ and so cannot determine the locus of our galaxies on the $M_{\mathrm{BH}^{-}}$ $\sigma$ plane. The HST observations, however, allowed us make accurate measurements of bulge luminosity and so find the locus of our galaxies on the $M_{\mathrm{BH}^{-}} \mathrm{L}_{\text {Bulge }}$ plane $(\S 4)$.

The layout of the paper is as follows. In section 2 we present observations and data reduction. The analysis is presented in section 3 and the results in section 4 . We then present a comprehensive discussion in section 5 and conclude in section 6 .

\section{Observations \& Data Reduction}

\subsection{Observations}

We observed with the ACS/HRC a sample of 10 NLS1s that do not appear to lie on the $M_{\mathrm{BH}}-\sigma$ relation (selected from Grupe \& Mathur 2004) and are close enough to achieve deconvolution of the bulge and the AGN. The details of the observations are given in Table 1. In Table 2 we present optical and X-ray properties of the sample galaxies that are generally used to define the NLS1 class; these are the $\operatorname{FWHM}(\mathrm{H} \beta)$, the X-ray power-law slope $\alpha_{X}$, the ratio of equivalent widths of FeII to $\mathrm{H} \beta$, and the Eddington luminosity ratio. We also list the BH mass estimates from Grupe \& Mathur (2004). In addition we quote the mean 
values of all the parameters with dispersion for the complete sample of soft X-ray selected NLS1s from Grupe et al. (2004). It can be clearly seen that the 10 NLS1s discussed in this paper are representative of the NLS1 population in general.

We used the F625W filter taking advantage of the fact that the HRC pixel size critically samples the PSF at $6300 \AA$. The large luminosity of the central AGN makes detecting the faint host galaxy underneath it difficult. To expand the dynamic range of the HRC, we took multiple exposures of each Seyfert galaxy: short exposures to well-measure the central core of the AGN's PSF, and longer exposures to pull out the surface brightness of the bulge (and disk) of the host galaxy.

\subsection{Data Reduction}

We used the standard ACS pipeline to reduce the data, with STSDAS in IRAF1. However, reduction of our observations was complicated due to the fact that all galaxies have a bright nucleus. This made it necessary to take several steps which are beyond the standard ACS pipeline2.

Pixel Repair: In half of our sample, one or more pixels in the core of the central AGN's PSF were saturated. In addition, electrons can spill over into pixels around a saturated pixel; this occurred most commonly to pixels in the same column as the saturated pixel, most likely during read-out. We therefore had to "repair" these pixels by replacing the value of its corrupted data number with a data number from a shorter exposure scaled by the exposure lengths. This process proved satisfactory for four of the five AGNs with saturated pixels, but in the case of IRASF12397 we found that even the shortest exposure was saturated in the very central pixel. However, a basic Gaussian fit indicated that any bleeding along the column was minimal, so we left this single pixel unrepaired in all of the exposures. The contribution of the pixel repair to the total profile uncertainty was found to be negligible compared to the much larger issue of the change in instrument focus and the effects that had on the PSF core/wings ratio (see below).

Cosmic Ray Rejection: Utilizing the same method as Bentz et al. (2009), we used the cosmic-ray rejection routine 'lacosmic' (van Dokkum 2001) to create a cosmic-ray mask of each of our images. We then removed any cosmic-ray flag from the central 11x11 pixels as

\footnotetext{
${ }^{1}$ http://iraf.noao.edu/

${ }^{2}$ see http://www.stsci.edu/hst/acs/documents/handbooks/currentDHB/acs_cover.html for details on ACS data reduction
} 
well as removing any flags associated with the Airy "ring". The PSF of the HRC does not produce a smooth Airy ring but instead produces a constellation of light "bulges" in place of a ring. This complicates cosmic-ray rejection algorithms and so we took manual control in this process to prevent the light in the constellated Airy "ring" from being damaged by the cosmic ray rejection routines. To ensure that no cosmic-ray had landed in the core of the AGN PSFs, we used a radial profile function (in imexamine within STSDAS) to look for discrepancies and then manually repaired affected pixels. The cosmic-ray mask was converted into a cosmic-ray list and the final cosmic-ray repair (both automated and manual) was handled in XVista 3 .

Registration: To combine our images as accurately as possible, we needed registration at the sub-pixel scale. We did this by centroiding the light from the AGN PSF of each exposure. The optimal method would involve averaging multiple point-sources across the chip and avoiding any extended source. However, we lacked any stars in the field of view of the majority of our targets. We found that centroiding only the AGN light was actually quite robust, for while there is indeed an extended source around it (the host galaxy), it is so faint that it does not affect the process. We used both qphot and imexamine to determine the centroid of the AGN PSF. In the majority of cases these two routines found the same centroid to within 0.05 of a pixel. Overall, the corrections to the preprogrammed dithering were under 0.07 pixels but could vary from an average of 0.04 pixels for TONS180 to 0.17 pixels for MRK478. The images then were coadded and the cosmic rays were removed, but did not (yet) undergo geometric undistortion.

We found that the library PSFs available for the ACS/HRC were insufficient in matching the nuclear light. Therefore we created a PSF template from a bright star in the field of RXJ2217 (at a projected distance of $36 \mathrm{kpc}$ so we are confident of no contamination) and performed a similar procedure to that discussed above. This star was (barely) unsaturated in the shortest exposures and we repaired the central pixels in the longer exposures. We then pulled a 201x201 pixel square around the star out of the image, detected and repaired the pixels hit by cosmic rays (of which there were few and none in the central core or Airy "ring") using lacosmic and XVista. We then fit the centroid of the PSF, and imshift to align the eight individual images, and coadded them in XVista. Finally, as the algorithm we used to deconvolve the components of this image (GALFIT; Peng et al. 2002) requires a sky-subtracted reference PSF, we fit the sky in the corners of the image away from the PSF halo and the PSF diffraction spikes and then subtracted the average scalar value from the image.

\footnotetext{
${ }^{3}$ http://ganymede.nmsu.edu/holtz/xvista/
} 
Finally, we fit each coadded (but not yet corrected for the geometric distortion of the HRC) image with this stellar PSF (representing the nuclear source), a deVaucouleurs profile (representing the central bulge), an exponential profile (representing the underlying disk), and a background sky. The purpose at this time was not to achieve a match to the galactic profile but rather to the nuclear source. In many cases manual control over the GALFIT routine had to be taken in order to provide the best match to the nuclear source. Because temperature fluctuations across HST change the focus of the instrument slightly ("breathing"), matches to both the core of the PSF and the outer regions was never perfect as each object was observed for one orbit. But as our purpose in this project is to best model the underlying galaxy, and these galaxies are relatively close, we concentrated upon matching the outer portions of the PSF. The metric used was the number of artifacts from the Airy ring and the diffraction spikes that remained upon subtraction of the PSF. Because the Airy ring in ACS/HRC has a beaded structure, it was rather easy to test for proper registration, PSF modeling, and PSF subtraction; a smoothed profile resulted in nine of the ten objects studied (please see figures 1 and 2 for the residuals of TONS180 for evidence of where our constructed PSF did not match as well as the other nine of our sample. TONS180 also has the brightest AGN in our sample which magnified this issue). The other major source of our ability to match the nuclear PSF was that this was all done before the correction for the geometric distortion of the HRC. As the geometric correction interpolates pixel positions, it naturally distorts the intrinsic PSF. The resultant PSF-subtracted images were then put back into PyDrizzle for the geometric correction to give us the final images from which to fit the galactic profiles (Figure 1).

\section{Analysis}

Using GALFIT, all ten targets were initially fit using three profiles: a fixed sky (previously derived from the corners of the image), and Sersic profiles, one started with $n=3.5$ and $R_{e}=1.0 \mathrm{kpc}$ (representing the bulge), and one with $n=1$ (fixed) and starting with $R_{e}=3.0 \mathrm{kpc}$ (representing the disk). An iterative process of examining the residuals and adding additional components was done for each target, testing each for significance (this also sometimes included data reduction components like residual PSFs or outright masking of the pixels associated with the PSF core for those galaxies whose PSF core/wings ratio was not as well fit by our model PSF). The expected features were found amongst our targets: non-traditional profiles, spiral arms, stellar bars, dust lanes, and (projected) nearby companions. In half the sample nearby companions (projected distance $\sim 10 \mathrm{kpc}$ and greater) were discovered but as these were not the focus of this study, the regions they occupied were masked out (in only one object did the companion overlap the visual extent of the target). 
Only one object was visually affected by asymmetric crossing dust lanes, but as these lanes were not easily excised by masking, manual control was taken of the fitting and the lanes were taken as residuals. Stellar bars of varying sizes were found (to varying degrees of certainty) in approximately $1 / 3$ to $1 / 2$ of the sample and the brightest were fit using a very boxy, high axis ratio profile with the proper position angle; please see the the discussion in $\S 4$ and the residuals column in Figure 1 for details. Spiral arms were generally taken into account by manual control in the last stages of the fitting. In fact, in all ten cases manual control was used to provide a comparison against the best fit made by the fitting algorithm to ensure against erroneous local minima.

The best fit profile parameters of all the targets are presented in Table 2. The bulges achieve an azimuthally integrated $\mathrm{S} / \mathrm{N}$ ratio at their $R_{e}$ between 150 to 360 . The final profile fits are shown in figures $3 \& 4$; the black line shows the data while the dotted and dashed blue lines show the disk and bulge components respectively. The red line is the sum of all components and shows that the bulge+disk profile fits the data well. There are small imperfections in two cases: RXJ2216.8 - 4451 and RXJ1702.5 + 3247. The galaxy RXJ2216.8 - 4451 is an asymmetrically dusty galaxy. The dust does not fall in lanes but rather in an irregular-shaped ring around the center at a distance of $\sim 2 \mathrm{kpc}$ (as seen in fig. 1). This results in the temporary flattening seen in its the radial profile from about 1.5 to $2.5 \mathrm{kpc}$ (Fig. 3). The galaxy RXJ1702.5 + 3247 is our furthest galaxy and also the one with the poorest initial deconvolution ( $(2.2)$. As can be seen in fig.4, there are significant artifacts left over from PSF subtraction (the two peaks correspond to the core and first Airy ring, as seen in fig. 2). The most likely cause are thermal fluctuations across the orbit ("breathing"). The disk profile is clearly seen but because the bulge profile may still be contaminated by the wings of the PSF, we caution that the reported bulge parameters of this galaxy are our most uncertain. The galaxy RX J1209.8+3247 only contains one component with $n=1$. This may be interpreted as a bulge-less disk or a pseudobulge with $n=1$ (see $\S 4$ ) and a fainter, undetected extended disk (see $\S 4$ for a discussion on pseudobulges). The presence of a supermassive black hole in this galaxy would be perhaps more surprising (and interesting) than the presence of a pseudobulge; pseudobulges with $n=1$ have been observed previously (Kormendy \& Kennicutt 2004 (KK04)) and so are AGNs in bulge-less galaxies (Araya et al. 2012).

One additional series of tests was done to ensure the quality of the fits. While in all cases but one, a bulge-like component and a disk-like component were found to be quite significant, comparisons were done between fits which (1) let the Sersic profiles of the bulge component entirely unconstrained, (2) limited the Sersic profile to $1 \leq n \leq 4$ and (3) fixed the Sersic profiles at $n=4$. Masking was also done on the central pixels which were within the limits of the core of the nuclear PSF as well to test the profile fitting. In the majority 
of cases, GALFIT naturally found Sersic profiles between 1.0 and 4.0. We discovered that

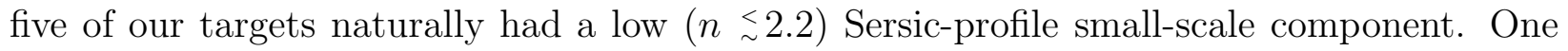
interpretation of this result is that these five galaxies have pseudobulges; we shall come back to whether these, and other galaxies with $n>2$, are real pseudobulges in $\S 4$. Of the remaining five, GALFIT found a Sersic index higher than $n=4.0$ in two cases. The other two were constrained to $n=4.0$, because GALFIT fit did not converge close to $n=4$. If left unconstrained, the "bulge" profile became steep and narrow, indistinguishable from a point source. One way of interpreting this might be that in these galaxies only one $(n=1)$ component is required and this may be a $n=1$ (pseudo)bulge with a faint. undetected disk, similar to what is observed for RXJ1209.8+3247. This will increase our pseudobulge candidates from 5 to 7 ; here we take a conservative approach and constrain $n=4$.

In general the sizes of the bulges we found are less than $2.0 \mathrm{kpc}$ (a cosmology of $\Omega_{m}=$ $0.3, \Omega_{\Lambda}=0.7$ was used to convert arcsec to $\mathrm{kpc}$, but all targets are relatively close by, at $z<0.17$ ). While the range of effective radii of bulges is within the normal range observed, we need to examine whether they are smaller than the true values, perhaps because of poor PSF subtraction. If this were the case then one would expect that the closest galaxies would have the "smallest" bulges while the furthest galaxies have the largest bulges because the PSF is a constant number of pixels but the plate scale changes by a factor of four. Such is not the case. In addition, the ratio of bulge effective radius to the visual extent of the PSF core has a median of $\sim 3$ (smallest two are 0.9 and 1.3 while the rest are larger than 2). So from this test, our bulge sizes appear to be real. Note also that Gadotti (2009) has shown that the structural properties of bulges can be reliably retrieved provided that the effective radius is larger than about $80 \%$ of the PSF HWHM; our measurements are significantly above this minimum requirement. The pseudobulge candidates (i.e., those with $n \lesssim 2$ ) ranged from 0.27 to $1.52 \mathrm{kpc}$ in size and the classical bulges (i.e., those with $n>2.5$ ) ranged from 0.37 to 1.34 kpc. Moreover, excess nuclear light left in the image would make the Sersic profile steeper, but the galaxies with the smallest ratio of $R_{e}$ to $R_{P S F}$ tended to be pseudobulge candidates instead (i.e. with flatter profiles). We thus conclude that our measurements of $R_{e}$ and $n$ do not suffer from artifacts of PSF subtraction.

Like the effective radii, the luminosities of these bulges also span more than a factor of ten. The images of these galaxies were taken in the F625W filter, more commonly known as SDSS $r^{\prime}$. The absolute $r^{\prime}$ magnitude of these bulges ranged from $-19.1 \mathrm{mag}$ to $-21.2 \mathrm{mag}$ with a median of about $-20.1 \mathrm{mag}$. The five galaxies with the pseudobulge candidates also had the four least-luminous bulges in our sample, again consistent with the $L-n$ relation. One consistency check would be to compare the HST and SDSS results for galaxies that have SDSS imaging. In those five cases, the absolute $r^{\prime}$ magnitude of our bulges were always less than the total absolute $r^{\prime}$ magnitude detected by SDSS, as expected. 
Our pseudobulge candidates do have properties consistent with the findings of other studies. Fisher \& Drory (2008) have shown that while classical bulges follow the structural parameters and the photometric projections of the fundamental plane, pseudobulges do not. While there is clear overlap in the structural parameters of bulges and pseudobulges in their sample, we note that the pseudobulge candidates of our sample occupy the same parameter space as that of pseudobulges of Fisher \& Drory (their figure 7); this provides a good consistency check. We have also tested whether redshift could be responsible for our low-Sersic-index bulges. While two of our pseudobulge candidates do have our lowest ratio of bulge effective radius to PSF core, the remainder all have ratios $\geq 2.5$. Among the pseudobulge candidates, one is the lowest redshift source and four are at high redshift, but two similarly high redshift sources also have high $n$ values. Thus our fitted parameters do not appear to be spurious on account of redshift.

\section{Results}

As pointed out by KK04, a pseudobulge is not an observational classification; it is a bulge made out of disk material by secular evolution. It is difficult to determine whether a bulge in a galaxy is a classical bulge (merger-driven) or a pseudobulge (secularly formed), but there are several indicators. Pseudobulges have one or more characteristics of disks: (1) flatter shapes; (2) larger ratios of ordered to random velocities; (3) small velocity dispersion $\sigma$ with respect to the Faber-Jackson relation between $\sigma$ and bulge luminosity; (4) spiral structure or nuclear bars in the "bulge" part of the light profile; (5) Sérsic index $n$ of the bulge surface brightness profile $\underset{\sim}{<}$; and (6) dominance of population I material (young stars, gas and dust) without a sign of a merger in progress (KK04). Pseudobulges do not follow the Kormendy relation (Kormendy 1977; a relation between surface brightness and effective radius); pseudobulges are fainter at a fixed effective radius (e.g. Fisher \& Drory 2008; Gadotti 2009).

We find that five of the ten galaxies have Sersic indices consistent with pseudobulges. All the pseudobulges in Gadotti (2008), irrespective of the Sersic index, have small bulge scale lengths with $R_{e}<2 \mathrm{kpc}$; all our galaxies have similarly small bulges. It is thus possible that some of our classical bulge candidates are also pseudobulges.

We can now find out where the NLS1 galaxies in our sample belong in terms of bulge properties and black hole mass. For the black hole mass estimate we use the standard single-

epoch procedure of using the $L_{5100 \AA}$ and $\operatorname{FWHM}(\mathrm{H} \beta)$ of these NLS1s (see Grupe \& Mathur 2004 for details). The most straightforward approach is to compare the black hole mass against the bulge luminosity, the so-called "Magorrian relation" (Magorrian et al. 1998). 
The most recent update on the Magorrian relation is published by Gültekin et al. (2009) who have determined bulge luminosity in the $V$ band. Our observations, however, are in the $r$-band, so we performed following color corrections. We use the average relation from Jester et al. (2005): $V=g-0.585 *(g-r)-0.01$. To obtain the $g-r$ color corrections we considered two options. One is to consider the bulge a part of the red sequence; we adopt the color sequence found in Bernadi et al. (2003) of $g-r=0.218-0.025 M_{r}$ (which gives an average color of $\sim 0.73$ ) for all our bulges with $n>1.5$. Pseudobulges, however, have been found to be still actively forming stars (KK04), so the $g-r$ color likely is more indicative of an average blue cloud galaxy, i.e. $g-r=0.5$. We show the result in Figure 5 taking into account both these color corrections; it is clear that the results are independent of the choice of color corrections. The figure shows that our NLS1s do not lie on the "Magorrian relation", but are actually below it. This confirms the results of Mathur and collaborators discussed in $§ 1$. Earlier we could not determine whether NLS1 bulges are generally overluminous for their black holes, or NLS1 black holes are undermassive for their bulges. We can rule out the former for at least part of our sample with pseudobulges; for the fixed size, pseudobulges are typically less luminous than classical bulges (Gadotti, 2009; Fisher \& Drory 2010). Thus it appears that the black holes in NLS1s are undermassive for their bulges.

We also compared our data with the "Magorrian relation" for broad-line AGNs (BLAGN) by Shen et al. (2008) and found consistent result, viz. our NLS1s do not follow the $M_{\mathrm{BH}^{-}}$ $\mathrm{L}_{\text {bulge }}$ relation of BLAGN. It should be noted that the Shen et al. luminosities are for the entire host galaxy (not just the bulge); taking this into account enhances the difference between NLS1s and BLAGN.

We have to keep in mind that our results may be affected by dust. As noted above our observations are in the HST $r$-band only, so we do not have any color information available for our sample. Naturally, the amount of extinction and reddening depends on the inclination of the galaxy, with face-on galaxies least affected. While one may determine the inclination for a disk by measuring the axial ratio, the same cannot be done for bulges, because we do not know their shape a priori. While it is not known whether the bulge and the disk of a galaxy have the same rotation axis, we can assume them to be the same for the time being. In Table 2 we have listed the axial ratios of the disks of our galaxies; most of them are pretty close to 1 , indicating they are close to face on, so the effect of dust is minimal. Graham \& Worley (2008) have given prescription for correcting the observed bulge magnitude for dust and for correcting the observed disk scale height and the disk central surface brightness; we will assume the same corrections for the bulge as well. Accordingly, our data points in Fig. 5 will move to right (higher luminosity) by a minimum of 0.56 (and more for more inclined galaxies), strengthening the result. 


\section{Discussion}

HST/ACS observations of our sample of 10 NLS1 galaxies have revealed that the bulge profiles range from "classical", i.e. near-deVaucouleurs, for five galaxies to "pseudo", i.e. near-exponential, for five galaxies. Thus at least half of our sample galaxies likely host pseudobulges. This is consistent with the results of Orban de Xivry et al. (2011) who find that NLS1s hosts preferentially have pseudobulges while BLS1 hosts preferentially have classical bulges.

$\mathrm{Hu}$ (2008) has shown that the $M_{\mathrm{BH}^{-}}-\sigma$ relation for pseudobulges is different from the relation for early-type bulges. Gadotti \& Kauffmann (2009) have also shown that the BHs in pseudobulges do not follow the $M_{\mathrm{BH}^{-}} \sigma$ relation of normal galaxies (and broad-line AGNs); they lie below the relation. Indeed, we find that the galaxies in our sample also lie below $M_{\mathrm{BH}}$-bulge luminosity relation. (We did not find the locus of our galaxies on the $M_{\mathrm{BH}^{-}}$ $\sigma$ plane, because we do not have direct measurements of $\sigma$; pseudobulges do not follow the fundamental plane either, so we cannot use the observed $\left\langle I_{e}>\right.$ and $R_{e}$ measurements to estimate $\sigma$ ). These results are fully consistent with the earlier results of Mathur \& collaborators $(\S 1)$ which showed that the locus of NLS1s on $M_{\mathrm{BH}^{-}}$-bulge relations is distinct from that of BLS1s and normal galaxies. Neither the black hole mass estimates nor the estimates of $\sigma$ based on narrow-line widths give a spurious result. Thus the black holes in NLS1 galaxies are truly undermassive for their bulges. They are, however, growing at a close-to-Eddington rate, so may reach the scaling relations of BLS1s eventually (Mathur 2000), provided they continue to accrete at the present rate. On the other hand, they may never reach the BLS1 scaling relations, especially if their BHs are growing slowly (Orban de Xivry et al. 2011).

As noted above, Gadotti (2008) has shown that pseudobulges do not lie on the fundamental plane. We can check whether our sample galaxies lie on the "photometric plane"

defined by Graham (2002). In figure 6, we have plotted the effective radius $\log \left(R_{e}\right)$ vs. $\log (n)+b<I_{e}>$ where $b=0.26$, the photometric plane. Points are our data and the solid line is the correlation found by Graham (2002) for E and S0 galaxies. The scatter around the correlation was found to be about $0.125 \mathrm{dex}$ in $\log R_{e}$ (dotted lines in Fig. 6). We see that four of our galaxies are off the line on this hyperplane, with offsets much larger than the scatter. Thus it seems that the pseudobulges do not lie on the photometric plane either. We note, however, that our observations are in the $r$-band, while the photometric plane is defined in the B-band. The color correction will move the points to the right by about 0.052, which will not affect our conclusion. Moreover, La Barbera et al. (2005) have shown that the photometric plane relation is independent of the waveband. Taking into account dust correction, the data points will move down (lower $R_{e}$ ) by a 0.045 and to the left (lower 
$<\mu>_{e}$ ) by 0.06 , effectively moving slightly away from the line, again strengthening the result. We also find that all 10 objects in our sample are offset from the projection of the fundamental plane of Barway \& Kembhavi (2007). Given that our sample is of late-type galaxies, perhaps a comparison with the photometric plane of dwarf ellipticals (Kourkchi et al. 2011) is more appropriate. We again find that our pseudobulge candidates lie off the relationship presented in Kourkchi et al.

Black hole masses in Seyfert galaxies are measured through reverberation mapping (Peterson 1993) when possible. The unknown geometry of the broad emission line region, however, leads to an uncertainty of the order of 0.5dex in measured masses, which is characterized by a factor $f$. Collin et al. (2006) estimated the value of $f$ under the assumption that all black holes lie on the $M_{\mathrm{BH}}-\sigma$ relation, and the observed scatter is solely due to the scatter in $f$. Because NLS1s were found to lie systematically below the $M_{\mathrm{BH}^{-}} \sigma$ relation, they derived a higher value of $f$ for NLS1s, compared to BLS1s, to move them back on the $M_{\mathrm{BH}}-\sigma$ relation. The results presented in this paper show that the Collin et al. result is not really valid for NLS1s because they occupy an intrinsically different locus on the $M_{\mathrm{BH}^{-}}$ $\sigma$ plane because their bulges are different. At this point it appears that calibrating the AGN mass scale with the $M_{\mathrm{BH}}$-bulge relationships (with $\sigma$ or $L$ ) will be either more difficult or more uncertain than hoped.

The $M_{\mathrm{BH}}$-bulge relations (with $\sigma$ or $L$ ) are also used to determine black hole masses in sources in which broad emission lines are not easily observed, e.g., blazars (Falomo et al. 2002), radio galaxies (Bettoni et al. 2003), bright cluster galaxies (Lauer et al. 2007; Batcheldor et al. 2007), and obscured AGNs (e.g. Greene et al. 2009). McLure \& Dunlop (2002) have also used the scaling relations to determine black hole masses of AGNs and suggest their use to determine black hole masses of high redshift galaxies. Because of the intrinsic scatter in these relations caused by a combination of factors such as the galaxy morphology, orientation and redshift evolution, application of scaling relations to determine BH masses may not be as straightforward as has been hoped.

As noted above, at least five of our NLS1 host galaxies have low-n bulges. If they are true pseudobulges, it has implications for black hole growth. Pseudobulges tend to show younger stellar populations as well as distinct structural and kinematic properties (rotational support), indicating different formation processes. While classical bulges are believed to have formed through mergers, pseudobulges are likely formed through secular evolution or long distance interactions. The pseudobulges are perhaps still in the formation process. It then follows that their nuclear supermassive black holes are also recently formed, and still growing. This is exactly what we had proposed for NLS1s, because they accrete at a higher Eddington rate compared to BLS1s (Mathur 2000). Thus the youth of NLS1s is supported 
by their growing black holes as well as their pseudobulge hosts. These results also suggest that there are different modes of black hole growth. At high redshift, the black holes appear to have grown quickly through merger-driven processes. These BHs are massive and inactive at the present epoch and reside in the centers of elliptical galaxies. In the galaxies hosting pseudobulges, the black holes are in the growth mode at the present epoch and the growth is triggered by secular processes. A secular slow, long, period of $\mathrm{BH}$ growth is also possible (Orban de Xivry et al. 2011). It should also be noted that the alternative, secular, mode of black hole growth is perhaps a dominant one at the present epoch. Weinzirl et al. (2009) have shown that about $70-75 \%$ of high-mass spirals contain pseudobulges, based on the values of Sersic index or the B/T ratio; Fisher \& Drory (2011) have also come to similar conclusion. Given that spirals outnumber ellipticals, it follows that the growth of black holes in most galaxies follows a secular track.

\section{Conclusions}

We observed ten NLS1 host galaxies with HST/ACS. We find that our sample AGNs lie below the "Magorrian relation" of normal galaxies and BLS1s, confirming earlier results of Mathur et al. We caution against using the $M_{\mathrm{BH}}$-bulge relations to determine black hole masses or to determine the geometry of the broad line region of AGNs. Image analysis revealed that five of them likely host pseudobulges. If they are true pseudobulges, it would imply that secular processes play important roles in galaxy evolution and black hole growth and that this alternative track of black hole-galaxy evolution may in fact be a dominant one.

Acknowledgment: This work is supported in part by the STScI grant HST-GO-10436 to SM and by the NSF grant AST-1008882 to BMP. 


\section{References:}

Abdo, A.A., et al. 2009, ApJ, 707L, 142

Adams, F.C., Graff, D.S., \& Richstone, D.O, 2001, ApJ, 551, L31

Aller, M.C., \& Richstone, D., 2002, AJ, 124, 3035

Barger, A.J., Cowie, L.L., Bautz, M.W., Brandt, W.N., et al., 2001, AJ, 122, 2177

Barway, S. \& Kembhavi, A. 2007, ApJ, 662, L67

Batcheldor, D., Marconi, A., Merritt, D., \& Axon, D., 2007, ApJ, 663, L85

Bentz, M. et al. 2006, ApJ, 644, 133

Bentz, M. et al. 2009, ApJ, 697, 160

Bernadi et al. 2003 ApJ 1251882

Bettoni, D., Falomo, R., Fasano, G., \& Govani, F. 2003, A\&A, 399, 869

Collin, S., Kawagichi, T., Peterson, B.M., Vestergaard, M. 2006, A\&A, 456, 75

Czerny, B. et al. 2001, MNRAS, 325, 865

Falomo, R., Kotilamen, J.K., \& Treves, A., 2002, ApJ, 569, L35

Ferrarese, L., \& Merritt, D., 2000, ApJ, 539, L9

Ferrarese, L., Pogge, R.W., Peterson, B.M., Merritt, D., Wandel, A., \& Joseph, C.L., 2001, ApJ, 555, L55

Fisher, D. B., \& Drory, N., 2008, AJ, 136, 773

Gadotti, D. A. 2009, MNRAS, 393, 1531

Gadotti, D. A., \& Kauffmann, G. 2009, MNRAS, 399, 621

Gebhardt, K., Bender, R., Bower, G., Dressler, A., Faber, S.M., et al., 2000a, A\&A, 539, L13

Gebhardt, K., Kormendy, J., Ho, L.C., Bender, R., Bower, G., et al., 2000b, ApJ, 543, L5

Graham, A. 2001, AJ, 121, 820

Graham, A. 2002, MNRAS, 334, 859

Graham, A., Onken, C. A., Athanassoula, E., \& Combes, F. 2010, arXiv:1007.3834 
Grupe, D., 2004, AJ, in press (April 2004), astro-ph/0401167

Grupe D. \& Mathur, S. 2004, ApJL, 606, 41

Greene, J., Zakamska, N., Liu, X., Barth, A., \& Ho, L. 2009, ApJ, 702, 441

Gültekin, K., et al. 2009, ApJ, 698, 198

Haehnelt, M., 2003, Classical and Quantum Gravity, 20, S31

Haehnelt, M.G., Natarajan, P., \& Rees, M.J., 1998, MNRAS, 300, 817

Häring, N. \& Rix, H.W. 2004, ApJ, 604L, 89

Hu, J., 2008, MNRAS, 386, 2242

Jester, S., et al. 2005, AJ, 130, 873

Jorgensen, I. et al. 1996, MNRAS, 280, 167

King, A., 2003, ApJL, 596, L27

Komossa, S. et al. 2006, AJ, 132, 531

Komossa, S. \& Xu, D., 2007, 667L, 33

Kormendy, J., 1977, ApJ, 218, 333

Kormendy, J. \& Kennicutt, R. C. 2004, ARAA, 42, 603

Kourkchi, E., Khosroshahi, H.G., Carter, D. \& Mobasher, B. 2011, MNRAS, 420, 2835

La Barbera, F. et al. 2004, A\&A, 425, 797

La Barbera, F. et al. 2008, ApJ, 689, 913

Lauer, T.R. et al. 2007, ApJ, 662, 808

Magorrian, I. et al. 1998, AJ, 115, 2285

Mathur, S., 2000a, MNRAS, 314L, 17

Mathur, S. 2000b, NewAR, 44, 469

Mathur, S., Kuraszkiewicz, J., \& Czerny, B., 2001, New Astronomy, Vol. 6, p321

Mathur, S. \& Grupe D. 2005a, ApJ, 633, 688

Mathur, S. \& Grupe D. 2005b, A\&A, 432, 463

McLure, R.J., \& Dunlop, J.S., 2002, MNRAS, 331, 795 
Merritt, D., \& Ferrarese, L., 2001, ApJ, 547, 140

Nikolajuk, M., Czerny, B., \& Gurynowicz, P., 2009, MNRAS, 394, 2141

Orban de Xivry et al. 2011, in preparation.

Osterbrock, D.E., \& Pogge, R.W., 1985, ApJ, 297, 166

Peng, C. Y., Ho, L. C., Impey, C. D. \& Rix, H. 2002, AJ, 124, 266

Peterson, B.M. 1993, PASP, 105, 247

Pounds, K.A., Done, C., \& Osborne, J., 1995, MNRAS,277, L5

Rodriguez-Ardila, A., Viegas, S.M., Pastoriza, M.G., \& Prato, L., 2002, ApJ, 565, 140

Shen, Van den Berk, Schneider, \& Hall 2008, AJ, 135928

Tremaine, S., Gebhardt, K., Bender, R., et al., 2003, ApJ, 574, 740

van Dokkum, P.G. 2001, PASP, 113, 1420

Wandel, A., 2002, ApJ, 565, 762

Watson, L., Mathur, S. \& Grupe, D. 2007, AJ, 133, 2435

Williams, R., Pogge, R.W., \& Mathur, S. 2002, AJ, 124, 3042

Williams, R., Mathur, S., \& Pogge, R.W. 2004, ApJ, 610, 737

Weinzirl, T., et al. 2009, ApJ, 696, 411

Woo, J. \& Urry, C.M. 2002, ApJ, 579, 530

Yu, Q., \& Tremaine, S., 2002, MNRAS, 335, 965

Yuan, W. et al. 2008, ApJ, 685, 801 
Table 1. Journal of Observations

\begin{tabular}{cccccc}
\hline \hline Object & Dataset & $\begin{array}{c}\text { Observation } \\
\text { Date }\end{array}$ & $\begin{array}{c}\text { Exposure } \\
\text { seconds }\end{array}$ & Redshift & $\begin{array}{c}\text { Scale } \\
{[\mathrm{kpc} / \text { arcsec }]}\end{array}$ \\
\hline MS 2254-36 & J96I10 & $2005-04-15$ & 2390 & 0.039 & 0.73 \\
IRAS F12397+3333 & J96I09 & $2005-01-16$ & 2464 & 0.044 & 0.84 \\
TONS 180 & J96I10 & $2005-08-18$ & 1528 & 0.062 & 1.13 \\
MRK 478 & J96I02 & $2005-04-30$ & 1542 & 0.077 & 1.44 \\
RXJ 2216.8-4451 & J96I03 & $2005-04-20$ & 2428 & 0.136 & 2.31 \\
MS 23409-1511 & J96I04 & $2005-05-06$ & 2150 & 0.137 & 2.32 \\
RXJ 1209.8+3247 & J96I05 & $2005-01-16$ & 2558 & 0.145 & 2.46 \\
RXJ 1117.1+6522 & J96I06 & $2005-04-18$ & 2544 & 0.147 & 2.48 \\
RXJ 2217.9-5941 & J96I07 & $2005-04-23$ & 2542 & 0.160 & 2.65 \\
RXJ 1702.5+3247 & J96I08 & $2005-04-23$ & 2370 & 0.164 & 2.70 \\
\hline
\end{tabular}


Table 2. Properties of the AGN observed by HST in comparison with the mean values of NLS1s

\begin{tabular}{lccccccc}
\hline \hline \multicolumn{1}{c}{ Object } & $\alpha_{2000}$ & $\delta_{2000}$ & FWHM $(\mathrm{H} \beta)^{1}$ & $\alpha_{X}{ }^{2}$ & Fe II/H $\beta^{3}$ & $L / L_{E d d}{ }^{4}$ & $M_{\mathrm{BH}}{ }^{5}$ \\
\hline Ton S 180 & 005720.2 & -222257 & $970 \pm 100$ & 1.89 & 0.90 & 6.30 & 7.1 \\
RX J1117.1+6522 & 111710.1 & +652207 & $1650 \pm 170$ & 1.89 & 0.99 & 0.40 & 21.0 \\
RX J1209.8+3217 & 120945.2 & +321702 & $1320 \pm 110$ & 3.18 & 1.09 & 1.45 & 5.4 \\
IRAS 12397+3333 & 124210.6 & +331703 & $1640 \pm 250$ & 2.02 & 1.79 & 0.76 & 4.5 \\
Mkn 478 & 144207.5 & +352623 & $1630 \pm 150$ & 2.08 & 0.97 & 0.15 & 26.9 \\
RX J1702.5+3247 & 170231.1 & +324720 & $1680 \pm 140$ & 2.13 & 0.98 & 1.86 & 21.7 \\
RX J2216.8-4451 & 221653.2 & -445157 & $1630 \pm 130$ & 2.48 & 1.13 & 1.78 & 16.7 \\
RX J2217.9-5941 & 221756.6 & -594130 & $1430 \pm 60$ & 2.69 & 0.96 & 1.02 & 12.4 \\
MS 2254-36 & 225739.0 & -365607 & $1530 \pm 120$ & 1.78 & 0.53 & 0.24 & 3.9 \\
MS 23409-1511 & 234328.6 & -145530 & $1030 \pm 100$ & 2.03 & 1.18 & 0.22 & 10.0 \\
Sample mean & & & 1451 & 2.2 & 1.05 & 1.4 & 12.96 \\
NLS1s mean & - & - & 1380 & 1.96 & 0.99 & 1.79 & 23.2 \\
NLS1s dispersion & - & - & 350 & 0.41 & 0.40 & 2.87 & 20.2 \\
\hline
\end{tabular}

${ }^{1} \mathrm{FWHM}(\mathrm{H} \beta)$ given in units of $\mathrm{km} \mathrm{s}^{-1}$ as listed in Grupe et al. 2004.

${ }^{2} \alpha_{X}$ taken from the ROSAT measurements as listed in Grupe et al. 2004.

${ }^{3} \mathrm{FeII} / \mathrm{H} \beta$ ratio given in Grupe et al. 2004.

${ }^{4}$ Eddington ratio $L / L_{E d d}$ derived from the Swift observations discussed in Grupe et al. 2010.

${ }^{5}$ Black hole masses derived from the virial relation as given by Kaspi et al. (2000). The values for the AGN here are in units of $10^{6} M_{\odot}$, listed in Grupe et al. 2010.. 
Table 3. Galaxy Properties

\begin{tabular}{|c|c|c|c|c|c|c|c|c|c|}
\hline \multirow[t]{2}{*}{ Object } & \multicolumn{3}{|c|}{ Bulge } & \multicolumn{4}{|c|}{ Disk } & \multirow{2}{*}{$\begin{array}{l}\log \mathrm{M}_{B H} \\
(5100 \AA)\end{array}$} & \multirow[t]{2}{*}{$<\mu>_{e, V}$} \\
\hline & $\mathrm{M}_{r}^{\prime}$ & $\mathrm{R}_{e}[\mathrm{kpc}]$ & $\mathrm{n}$ & $\mathrm{M}_{r}^{\prime}$ & $\mathrm{R}_{e}[\mathrm{kpc}]$ & $\mathrm{n}$ & $\mathrm{b} / \mathrm{a}$ & & \\
\hline MS 2254-36 & -19.1 & 0.27 & 2.12 & -19.7 & 2.0 & 1 & 0.77 & 6.60 & 16.9 \\
\hline IRASF $12397+3333$ & -20.2 & 0.88 & 3.45 & -19.8 & 3.6 & 1 & 0.42 & 6.67 & 18.4 \\
\hline TON S180 & -20.1 & 1.90 & 5.45 & -20.1 & 4.5 & 1 & 0.85 & 6.85 & 20.2 \\
\hline MRK 478 & -21.2 & 0.95 & $4.00^{\mathrm{a}}$ & -21.2 & 5.9 & 1 & 0.96 & 7.44 & 17.7 \\
\hline RX J2216.8-4451 & -21.1 & 1.34 & $4.00^{\mathrm{a}}$ & -21.1 & 3.85 & 1 & 0.94 & 7.23 & 18.8 \\
\hline MS 23409-1511 & -20.7 & 0.37 & 5.31 & -20.4 & 5.5 & 1 & 0.93 & 7.01 & 16.1 \\
\hline RX J1209.8+3247 & -19.8 & 1.12 & $1.00^{\mathrm{b}}$ & $\ldots$ & $\ldots$ & $\ldots$ & $\ldots$ & 6.75 & 19.6 \\
\hline RX J1117.1+6522 & -19.7 & 0.45 & 0.62 & -21.4 & 5.40 & 1 & 0.94 & 7.33 & 17.7 \\
\hline RX J2217.9-5941 & -19.6 & 0.33 & 0.78 & -21.1 & 3.0 & 1 & 0.86 & 7.10 & 17.2 \\
\hline RX J1702.5+3247 & -19.8 & 1.52 & $0.69^{\mathrm{c}}$ & -20.8 & 6.7 & 1 & 0.91 & 7.34 & 20.3 \\
\hline
\end{tabular}

${ }^{\text {a} F o r c e d ~ S e r s i c ~ p r o f i l e ~} 1 \leq \mathrm{n} \leq 4$

${ }^{\mathrm{b}}$ Only one component necessary

${ }^{\mathrm{c}}$ Detection of Bulge marginal 


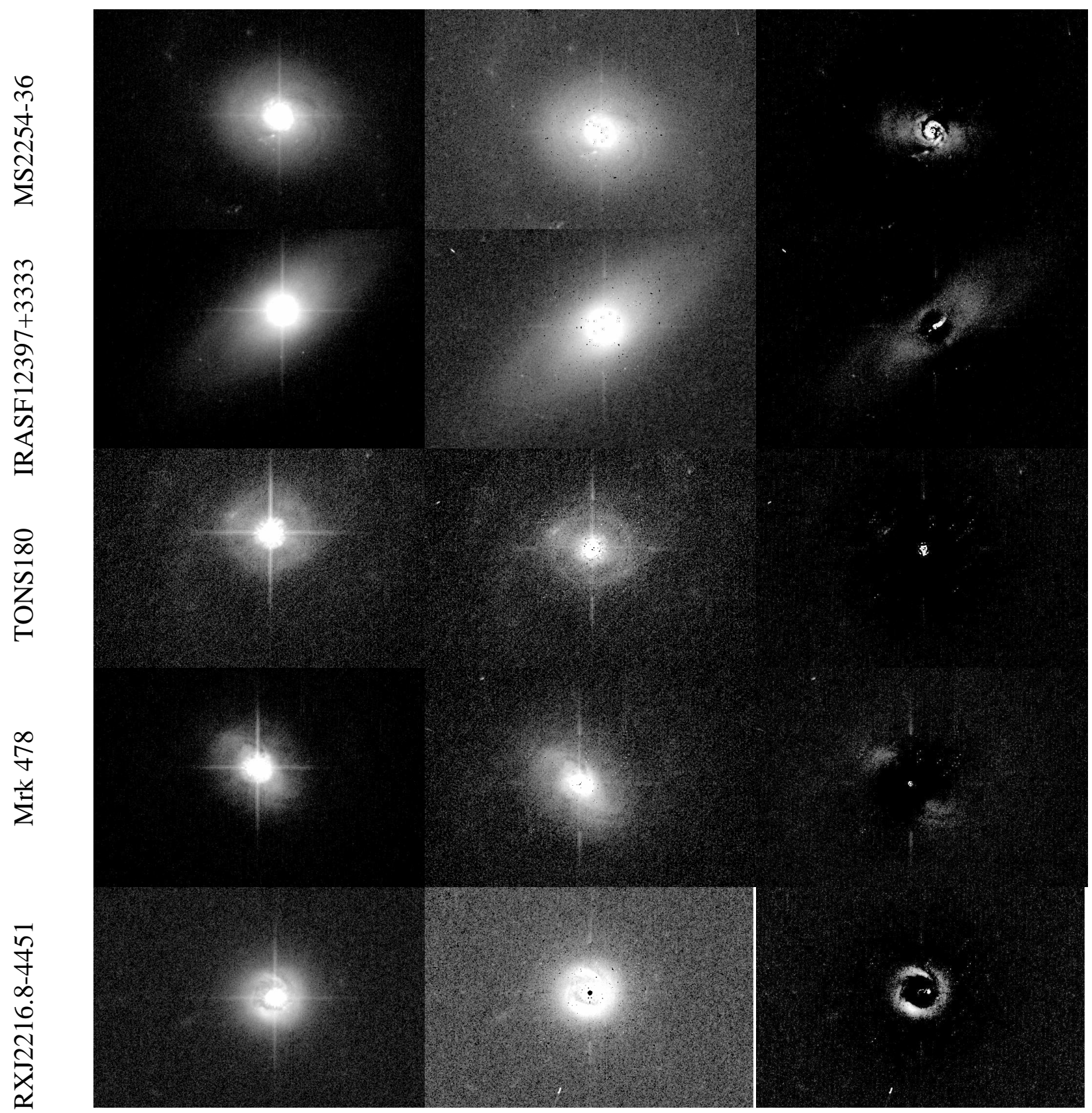

Fig. 1. - ACS images of our sample galaxies. The left column shows the observed images, and the middle column shows images after nuclear point source subtraction. These images were fitted with bulge and disk profiles; the residuals to the fit are shown in the right column. Several images show structures such as bars and spiral arms (see text). 


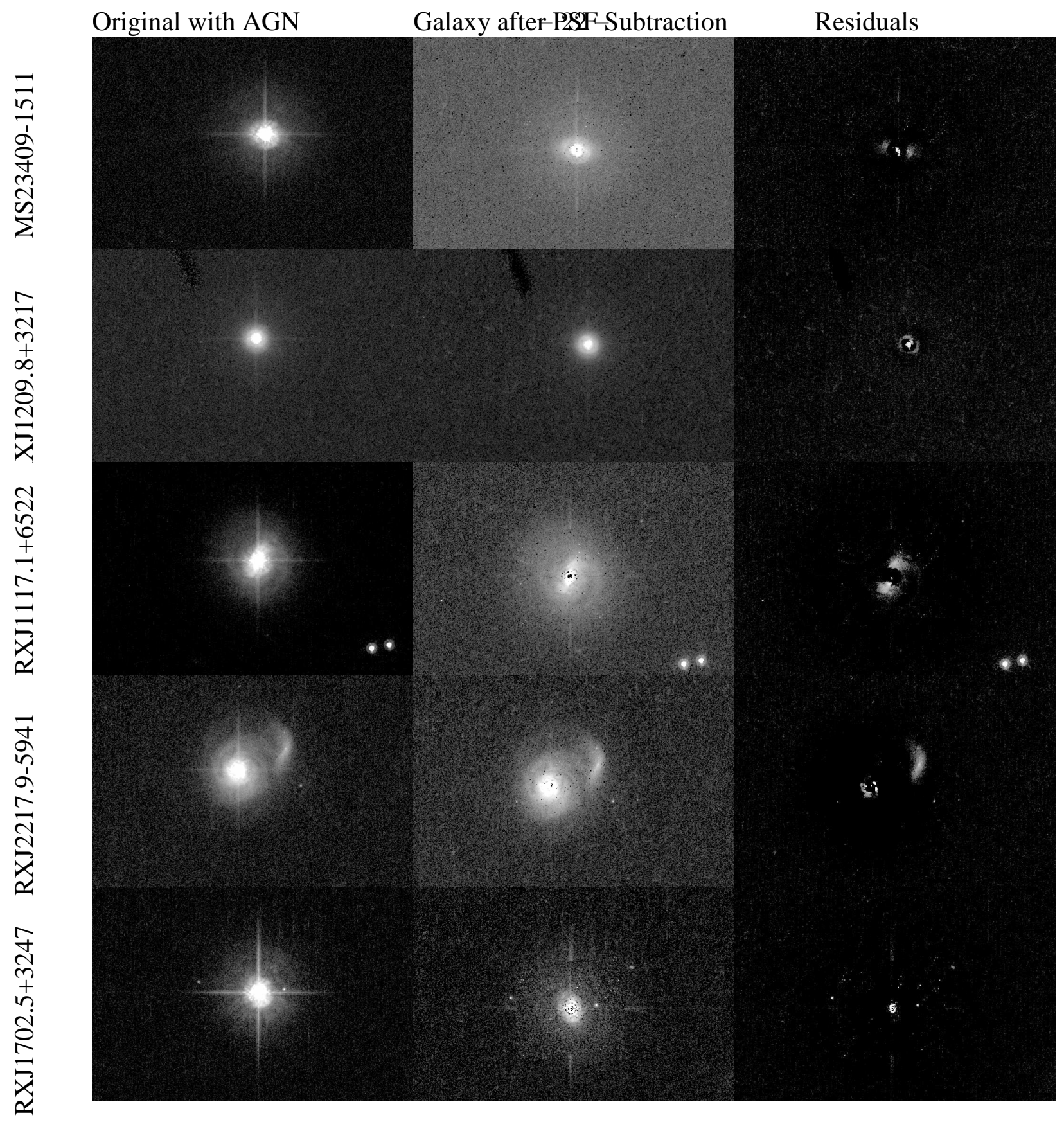

Fig. 2.- Figure 1, continued. 


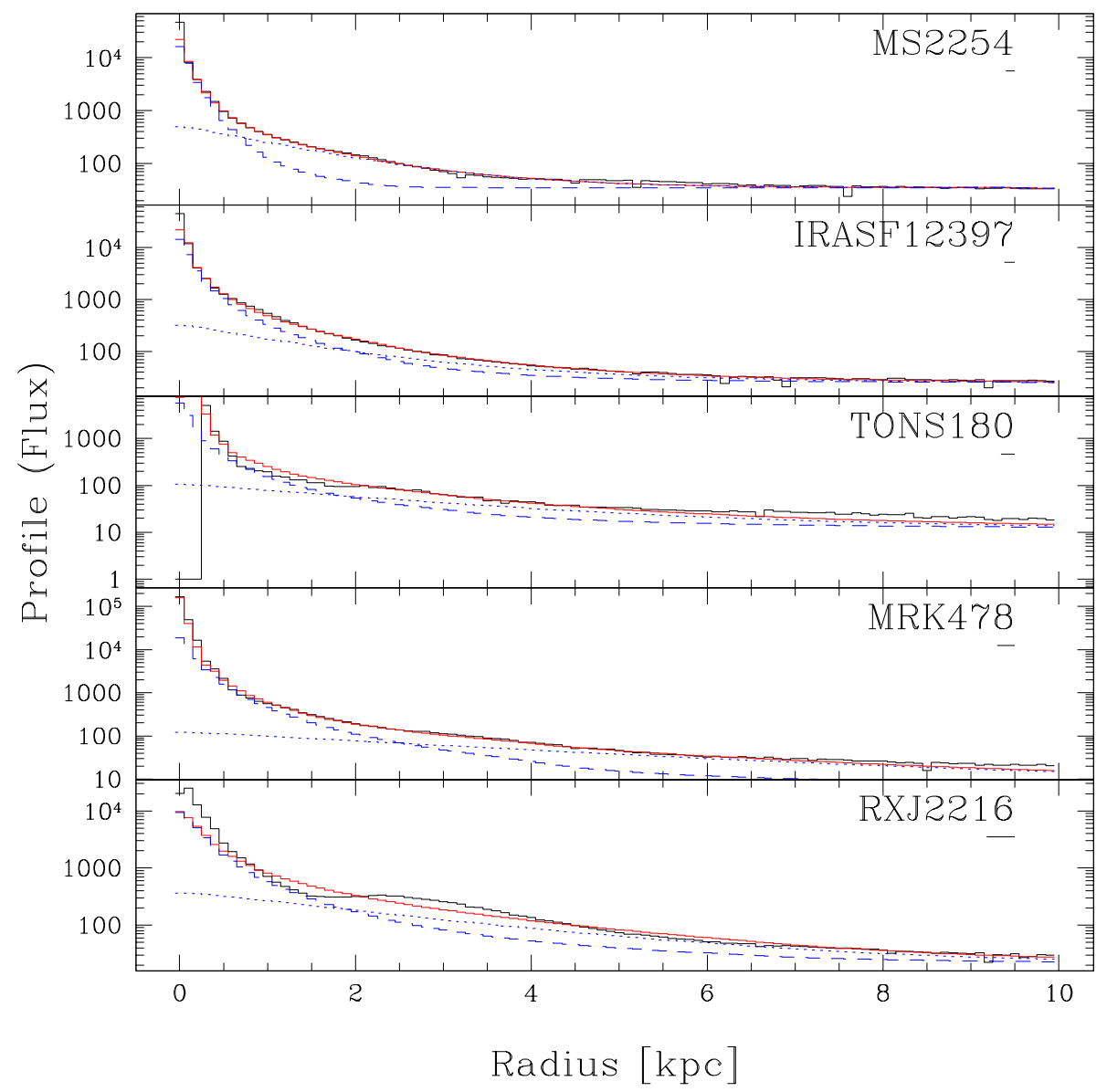

Fig. 3.- Radial profiles of the sample galaxies. The dotted blue line shows the disk component, while the dashed blue line shows the bulge component and the solid red line is the sum of the two (the sky is included in all). The black line is the data. The short horizontal bar on the upper right corner (below the galaxy name) shows the size of the PSF core (5 pixels). This shows that the galaxies are well sampled and are well fit by the bulge+disk profile. 


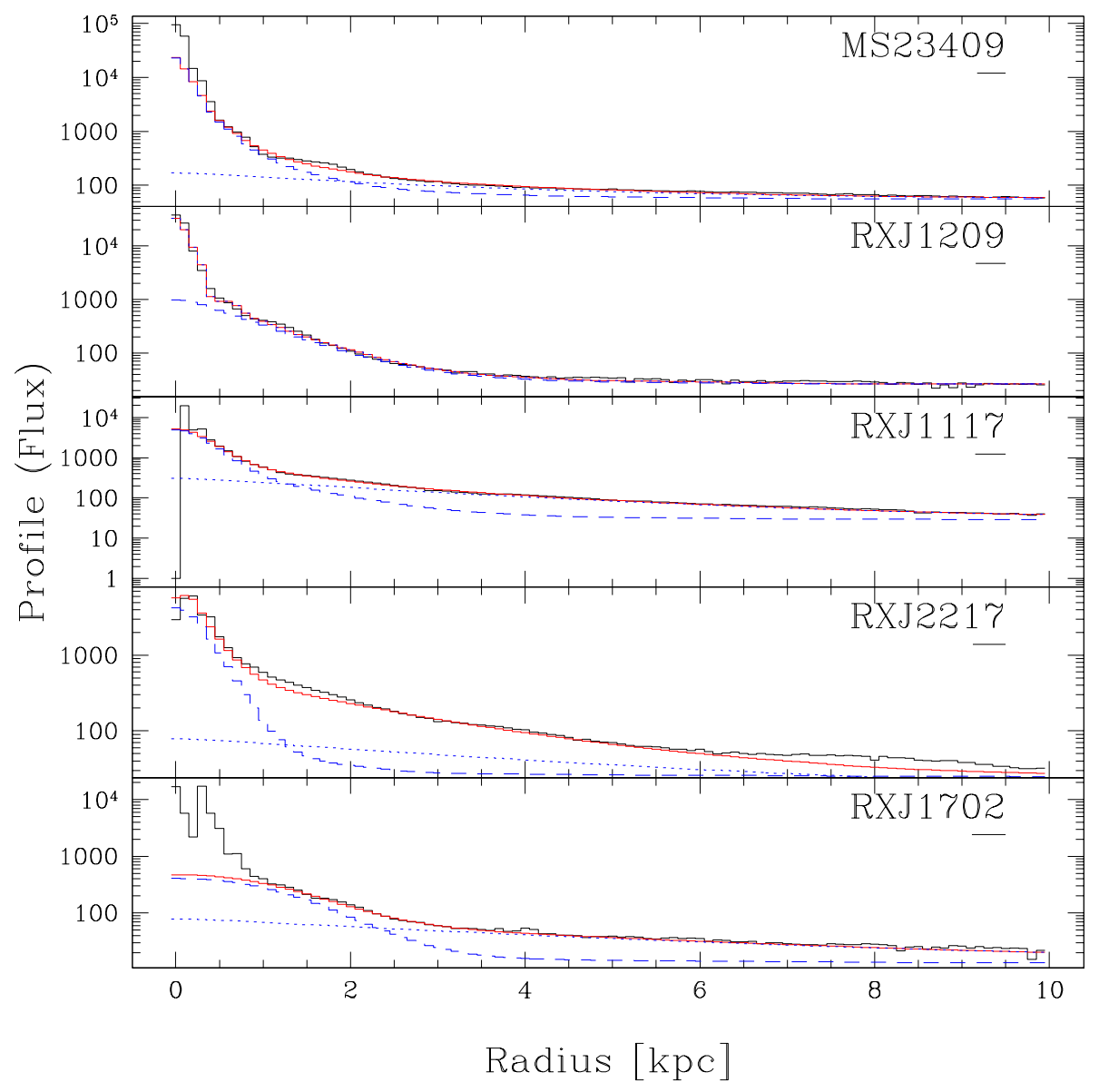

Fig. 4.- Figure 3, continued. 


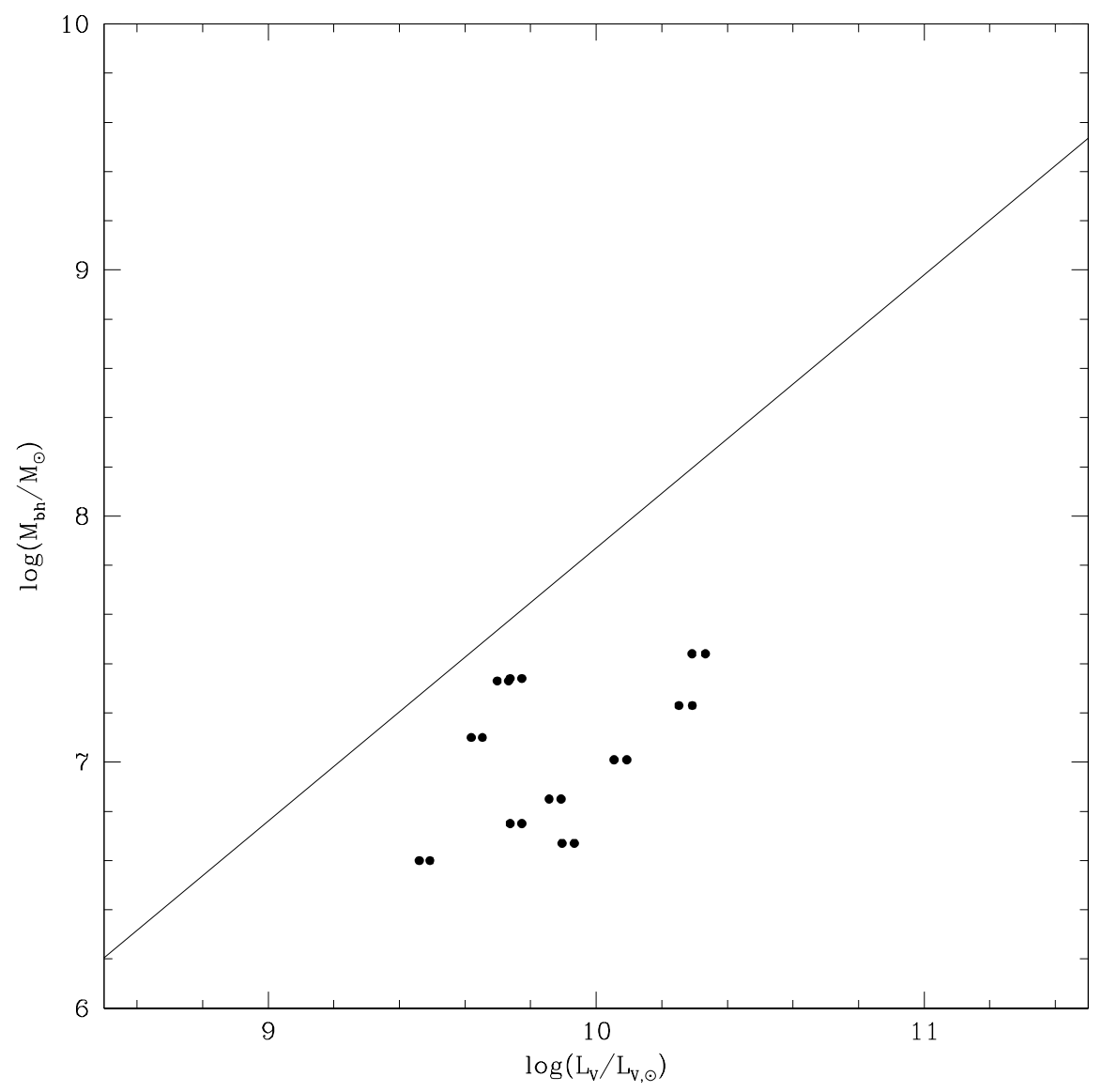

Fig. 5. - The black hole mass vs. the host bulge luminosity for our sample of NLS1s. For each galaxy there are two points joined by a bar corresponding to two different assumptions about the color corrections. The line is the black hole mass-bulge luminosity relation from Gültekin et al. 2009. It is clear that our sample galaxies do not follow the Gültekin et al, relation, but lie below that relation. The measurement error on $\log L_{V} / L_{\odot}$ is smaller than the color correction shown. The error on black hole masses estimates from single epoch spectra is generally believed to be about 0.3 dex. 


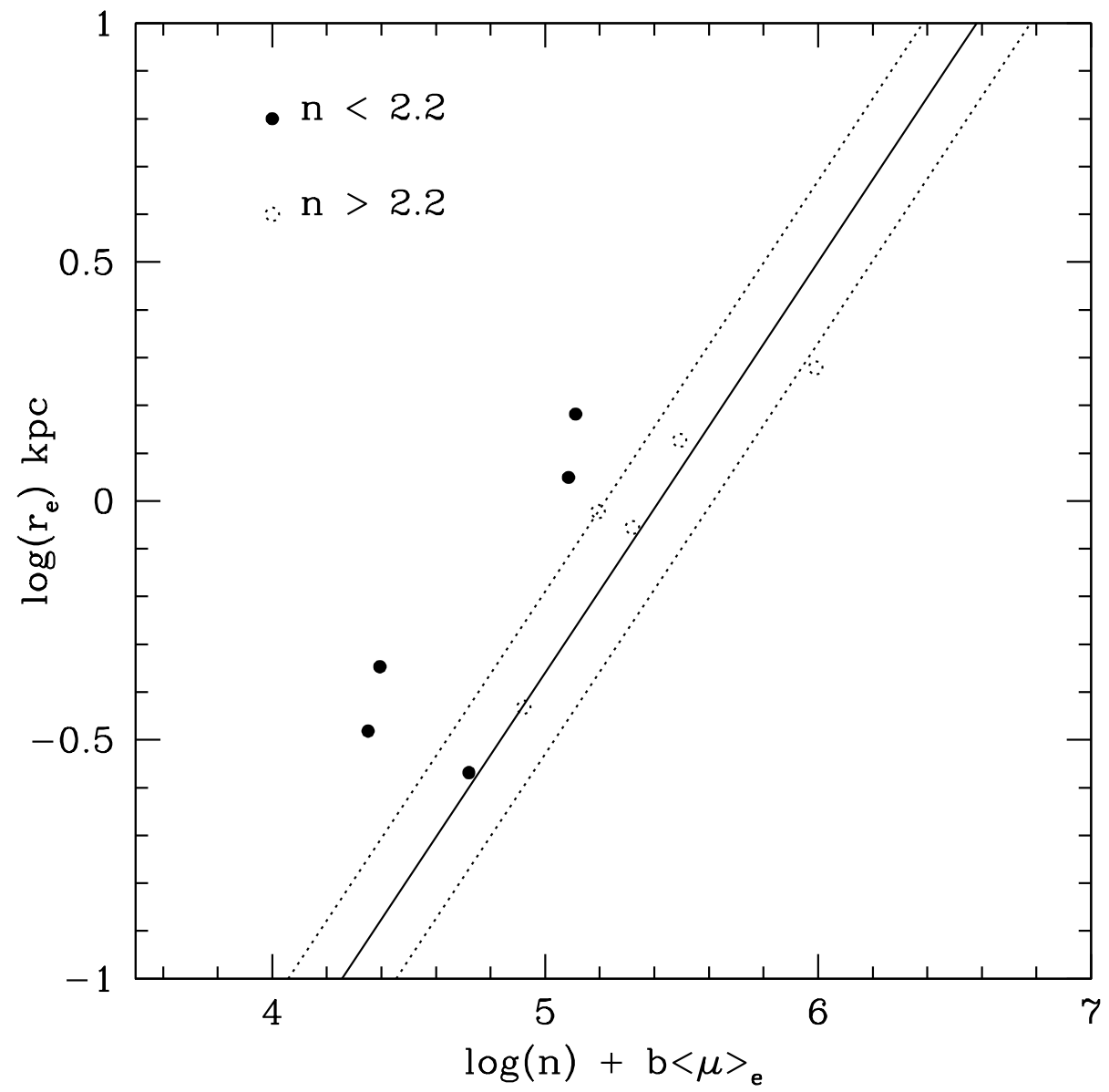

Fig. 6.- Photometric plane. The solid line is the best fit found for E \& S0 galaxies by Graham (2002). The points are our data. It appears that the pseudobulges do not lie on the photometric plane relation, just as they do not lie on the fundamental plane. 